\title{
Cannabis terapéutico y COVID-19: entre el oportunismo y la infoxicación
}

\section{Therapeutic Cannabis and COVID-19: between opportunism and infoxication}

\author{
Francisco Pascual Pastor*, Manuel Isorna Folgar**, Nelson Carvalho***, \\ Félix Carvalho****, Francisco Arias Horcajadas*****. \\ * Presidente Socidrogalcohol. Asesor de CAARFE. Investigador Grupo Prevengo UMH. \\ Coordinador UCA-Alcoi. Grupo Ibero Ciência. \\ ** Universidad de Vigo. Grupo PS1. Ayuntamiento de Catoira. Grupo EVICT. Grupo Ibero Ciência. \\ *** Unidade Operacional de Intervenção em Comportamentos Aditivos e Dependências - IASAÚDE - IP-RAM. \\ Grupo Ibero Ciência. \\ **** UCIBIO, REQUIMTE, Laboratório de Toxicología, Faculdade de Farmácia da Universidade do Porto. \\ Portugal. Grupo Ibero Ciência. \\ ***** Programa de Alcohol y Patología Dual. Hospital Doce de Octubre, Madrid. Grupo Ibero Ciência.
}

\section{La pandemia y el consumo de las drogas}

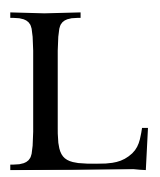

a pandemia por coronavirus (COVID-19) registrada en los últimos meses ha causado una alarma social sin precedentes a nivel mundial. Se estima que las reacciones de ansiedad, preocupación o miedo aumentarán en la población debido a su carácter desconocido, a la incertidumbre que lo rodea, a lo que debemos de añadir las medidas de distanciamiento social y confinamiento derivadas del estado de alarma (García-Álvarez, de la Fuente-Tomás, Sáiz, García-Portilla y Bobes, 2020).

Ante el cambio tan sustancial que supone este escenario en nuestros hábitos diarios a nivel de dinámicas familiares, a nivel laboral (teletrabajo, pérdidas empleo, expedientes de regulación de empleo, etc.), en las relaciones sociales y/o en la ocupación del tiempo de ocio, la población se va adaptando como buenamente puede a esta nueva situación. De esta forma observamos como numerosas personas realizan actividades deportivas y culturales en su propia casa, se mantienen en contacto con sus amistades y familiares a través de los dispositivos que nos ofrece la tecno- logía, utilizan los teléfonos que prestan ayuda psicológica varias ONG y profesionales sanitarios. Sin embargo, otras actividades como el consumo de alcohol, tabaco, psicofármacos y el cannabis podrían incrementarse no solo como forma de distracción o estrategia de evitación conductual, sino también como consecuencia del estrés, ansiedad o los síntomas depresivos que están experimentando (García-Álvarez et al., 2020).

Por otro lado, las personas consumidoras de substancias psicotrópicas también se enfrentan a desafíos únicos, principalmente los de drogas ilegales debido a la dificultad para adquirir las sustancias durante el confinamiento, pero también a nivel de vulnerabilidad ya que aquellas que fuman o vaporizan productos derivados del cannabis (también tabaco), las que dependen de los opioides y el uso de metanfetamina también pueden tener un mayor riesgo de complicaciones debido a los efectos respiratorios y pulmonares de la infección por SARS-CoV2 (Dunlop et al., 2020). En comparación con la población general, las personas con trastornos por consumo de sustancias presentan una mayor carga de enfermedades comórbidas (Schulte y Hser, 
2013; Wu, Ghitza, Zhu, Spratt, Swartz y Mannelli, 2018). Al mismo tiempo, es probable que las personas que consumen drogas sean más vulnerables durante la epidemia de COVID-19, debido principalmente a una peor alfabetización en temas de salud, al estigma o discriminación social hacia este colectivo, y que una parte importante de los consumidores suelen priorizar el consumo de drogas por encima de otros problemas de salud (Dunlop et al., 2020).

\section{Aprovechando las circunstancias problemáticas}

Desde hace décadas, pero con especial énfasis en los últimos tiempos, las proliferaciones de noticias falsas sobre las propiedades presuntamente sanadoras del cannabis se han visto incrementadas. Incluso a lo largo del confinamiento ciertos colectivos pretenden que el cannabis y sus productos sean considerados como «bien de primera necesidad, dado su extenso uso terapéutico", de manera que los clubes y asociaciones que la dispensan puedan seguir activos durante la pandemia (García, 2020). Pero ¿Qué hay detrás de esto? ¿Cómo hemos llegado a este estado de opinión favorable hacia su consumo en una parte importante de la ciudadanía? La permisividad de publicidad engañosa distribuida en las tiendas físicas y online, la difusión en ferias, foros, webs y las propias manifestaciones vertidas por: los consumidores en clubes cannábicos, grupos de presión, defensores de su legalización en foros especializados e incluso ciertos políticos (independientemente de su ideología) han contribuido a crear este estado de opinión. Para ello han utilizado estrategias muy cuestionables pero efectivas como: comparaciones con países y legislaciones diferentes, opiniones de los propios consumidores y sobre todo referenciando estudios más que discutibles por su escasa validez científica y con sesgos evidentes. Conviene subrayar, tal y como recoge la NACEM (Asociación Nacional de Ciencias, Ingeniería y Medicina de los Estados Unidos, 2017) en su informe, que en la mayoría de las ocasiones las conclusiones a las que llegan ciertos estudios o investigaciones no se sintetizan, traducen o comunican de manera adecuada a los encargados de formular políticas, al personal sanitario y a los profesionales de entidades asociadas. $Y$ es que existe una diferencia con otras sustancias, como el alcohol y el tabaco, pues el riesgo inherente a su consumo no puede determinarse ya que no existen estándares aceptados para un «consumo seguro», ni tampoco dosis adecuadas disponibles para orientar a los consumidores. De hecho, en la actualidad existe una conformidad científica en reconocer que las repercusiones negativas de su consumo a nivel físico, cognitivo, emocional y conductual aumentan entre aquellos que comienzan a consumirlo en plena adolescencia, mostrando en líneas generales una mayor probabilidad de desarrollar un patrón de consumo de riesgo y un repertorio de consumo más perjudicial (Filbey,
Mc-Queeny, DeWitt y Mishra, 2015; Rial, Burkhart, Isorna, Barreiro, Varela y Golpe, 2019). Además, el «consumo recreativo» o «terapéutico» posiblemente se ve afectado por una gran variabilidad individual.

Como explicamos anteriormente, la ciudadanía en su conjunto está viviendo una situación sanitaria sin precedentes. El confinamiento está generando en parte de la población una cierta incertidumbre y en algunas personas un malestar físico y psicológico destacable. En estos días hemos escuchado en innumerables ocasiones, de forma reiterativa y por diversos medios, que el cannabis puede aliviar cierta sintomatología negativa debido a sus potenciales beneficios terapéuticos (Ej. relaja, incrementa el apetito, calma la ansiedad, alivia el dolor y te evade de la realidad) e incluso puede combatir el COVID-19 o al menos minimizar su sintomatología. Ese parece ser el planteamiento, hacer llegar a la población el mismo mensaje que ha recibido de otras patologías y que asocia cannabis con terapia curativa.

Precisamente en estos momentos y sirviéndose de la pandemia han aparecido más publicaciones atribuyendo distintas propiedades potencialmente terapéuticas de algunos cannabinoides, no del cannabis propiamente dicho, ique no es lo mismo! Aunque la tendencia actual, en aquellos que defienden el uso del «cannabis terapéutico», es huir de disertar sobre los efectos del tetrahidrocannabinol (THC) y abogan principalmente por los efectos del cannabidiol (CBD), cannabinol (CBN) y del cannabigerol (CBG). Pero no todos, porque hay colectivos y grupos de presión que sigue alabando y manifestando las propiedades de los «verdes-Joint» o «porros-spliff», sin excepciones ni fracciones, demandando la legalización y abogan por el autocultivo para el auto consumo (Confac, 2020; García, 2020).

A lo largo de este periodo de confinamiento ha habido un gran despliegue mediático sobre el cannabis y su potencialidad para «tratar» la tormenta de citoquinas asociada a COVID-19, una desregulación inmune fatal que cursa durante el curso de la enfermedad. Si bien hay una escasez de datos en humanos sobre infecciones virales agudas y cannabinoides, los estudios que se han realizado hasta el momento actual arrojan un halo de esperanza sobre su potencial papel en la supresión inmune en la enfermedad del COVID-19, sin embargo, cabe destacar que los estudios realizados han sido experimentos in vitro e in vivo (Sexton, 2020).

La propia Organización Mundial de la Salud advierte sobre la importante amenaza que acompaña a la COVID-19: «la infodemia» o sobreabundancia de información de rumores, bulos y datos falsos que propagan ciertos grupos con intereses manifiestos que pretenden desinformar y confundir a la ciudadanía. A continuación, se presentan a modo de ejemplo algunos de los titulares publicados que relacionan cannabis con COVID-19:

El cannabis no cura el coronavirus, pero puede ayudar a aliviar ciertos síntomas». “iBuenas noticias! El Cannabis puede 
curar el COVID-19»; "Nueva York ordena detener la venta de un producto de cannabis como cura de COVID-19»; «Cierran dispensario de marihuana en Arizona por promover falsa «cura» para el coronavirus»; "Compañia canadiense investigará efectos del cannabis en síntomas de COVID-19»; «10 propuestas cannábicas a realizar en tiempos de coronavirus»; «La marihuana como servicio esencial»; «Investigadores encuentran en la marihuana la posible cura al coronavirus.

Por lo tanto, vistas y analizadas ciertas noticias o «fake news» que han aparecido principalmente en internet y las redes sociales relacionadas con el cannabis y el COVID, nos vemos en la obligación moral y científica de combatir estos bulos y contribuir a que la ciudadanía disponga de información veraz, fiable y respaldada por la evidencia científica.

Cabe señalar primeramente la opinión de Nora Volkow (2020), directora del Instituto Nacional sobre el Abuso de Drogas (NIDA), al respecto en el contexto de la pandemia de COVID:

«El riesgo de gravedad e incluso de muerte producida por el COVID-19 aumenta con la edad avanzada pero también se concentra entre quienes están inmunocomprometidos o tienen su salud comprometida con distintas afecciones como diabetes, cáncer, cardiopatías y enfermedades respiratorias. Muchos de estos últimos surgen de fumar y, por lo tanto, puede aumentar el riesgo de muerte y enfermedad entre fumadores (tabaco y/o cannabis)».

Similar al consumo de tabaco, el consumo de cannabis puede provocar efectos a largo plazo del aumento de la tos, la producción de esputo y las sibilancias, junto con enfermedades de las vías respiratorias como la bronquitis crónica y la disminución de la función pulmonar (Owen, Sutter y Albertson, 2014; Tashkin, 2013). Además, los datos obtenidos de fumadores de cannabis adultos sanos demostraron una reducción global en la producción de citoquinas (McCoy, 2016; Sexton et al., 2014). De igual forma, el uso de «vaper» (dispositivos electrónicos para la vaporización de nicotina y que tras su manipulación se utilizan para consumir también de THC y de CBD, puede presentar un riesgo aún mayor a través de la concentración, la adulteración o la contaminación de los extractos. La concentración de cannabinoides y terpenos se puede aumentar entre 3.2 y 4 veces y entre 2.7 y 8.9 veces, respectivamente (dependiendo del proceso de extracción y la estructura del terpeno), y esta forma tan concentrada puede también contribuir a potenciar los síntomas y la disfunción respiratoria (Sexton, Shelton, Haley y West, 2018). Evitar fumar cannabis y vapear concentrados de THC y CBD es particularmente relevante para la salud pulmonar a la sombra del COVID-19. Estos métodos de administración pueden disminuir la eficacia del sistema respiratorio para responder a la infección y, por lo tanto, aumentar el riesgo de progresión rápida a hipoxemia (Sexton, 2020).

Cabe destacar la peligrosidad del consumo de cannabis en cachimba (pipas de agua, sisha, argille o hookah) ya que permite que las inhalaciones envíen más humo a los pulmones y por tanto más THC (y nicotina), lo que amplifica el efecto del cannabis debido principalmente a su efecto broncodilatador (Tetrault, Crothers, Moore, Mehra, Concato y Fiellin, 2007). Es una característica habitual de los fumadores de cannabis inhalar profundamente y contener la respiración para lograr la máxima absorción de THC en los pulmones (Hall, Degenhardt y Teesson, 2009). A ello hay que añadir la combustión del carbón en la cachimba que produce: sustancias cancerígenas, metales pesados, hidrocarburos aromáticos policíclicos y diversas toxinas, todas estas sustancias han sido relacionadas con enfermedades pulmonares, cardiacas y cáncer (Etemadi et al., 2017). Al ser una práctica social en grupo, se comparten las boquillas y la manguera de la cachimba; esta práctica constituye un problema sanitario, ya que aumenta el riesgo de transmisión de enfermedades respiratorias como la tuberculosis o de virus como el de la hepatitis o herpes (Mycobacterium tuberculosis, Helicobacter pylori, Hepatitis C virus, Epstein-Barr, Herpes simplex, Virus respiratorio y Aspergillus) debido principalmente a la humedad existente y a que en muchas ocasiones no se cambia el agua de la cachimba hasta varias sesiones lo que crea un caldo de cultivo de bacterias que puede ser muy perjudicial para la salud (Galindo, González, Espigares y Moreno, 2019). La infección es causada por compartir la boquilla entre varias personas lo que actúa como vehículo de transmisión ya que cualquiera de esas personas puede tener gingivitis u otro tipo de lesión en la cavidad oral y eso los hace más propensos a transmitir o ser infectados (Blachman-Braun, Del Mazo-Rodríguez, López-Sámano y Buendía-Roldán, 2014). Precisamente coincide con las vías de trasmisión del COVID, gotículas al respirar, aerosoles y superficies infectadas compartidas, con lo que la contraindicación de su consumo es absoluta.

Al mismo tiempo cabe destacar que el servicio de farmacología y toxicología clínica del hospital universitario de Génova (Agoritsas et al., 2020) ha realizado una evaluación farmacológica del uso de los cannabinoides en las personas infectadas por SARS-CoV-2, partiendo de la base que el uso de los fitocannabinoides en los modelos experimentales revelan resultados contradictorios y señalan que un consumo cotidiano de cannabis a largo plazo está asociado a una progresión fibrótica en caso de hepatitis $\mathrm{C}$, conduciendo a una replicación viral de la hepatitis, y el THC está asociado también a un aumento de la replicación viral en el VIH e incrementa la carga viral en la Gripe (Influenza), en modelos animales. Ante estas premisas, el riesgo del consumo de cannabis en la COVID-19 puede ser muy importante, y subrayan que en estos momentos no hay ningún estudio con 
base científica que recomiende el consumo de cannabis y sus derivados en caso de COVID. Por lo que entendemos que cualquier anuncio sobre su presunta utilidad carece de evidencia científica. Por el contrario, la falsa afirmación de que ciertos cannabinoides «refuerzan el sistema inmunitario» o la falacia sobre los potenciales efectos antivirales del CBD son refutados por la evidencia farmacológica y clínica actual que manifiesta que el CBD (y el THC) podrían disminuir la capacidad de combatir infecciones; lo cual contrasta con sus posibles usos clínicos como antiinflamatorio Este riesgo puede ser incluso mayor en las infecciones virales y respiratorias (Brown, 2020). Por lo tanto, es necesario recomendar a usuarios y personal sanitario evitar el consumo de CBD y otros cannabinoides durante esta pandemia, a menos que sea por prescripción facultativa (por ejemplo, convulsiones, cáncer, dolor crónico).

Así pues, Satre, Hirschtritt, Silverberg y Sterling (2020) proponen que, en el contexto de la pandemia, el personal sanitario y proveedores deben aconsejar a los consumidores adultos mayores de 60 años de cannabis, que deben abandonar el consumo fumado y el vapeo o al menos reemplazarlo por formas comestibles; medidas que, de aplicarse, probablemente mejoren las posibilidades de supervivencia de COVID-19. También se les debe alertar de los efectos adversos incluidas las caídas, la ansiedad y la dependencia. Del mismo modo a los fumadores de tabaco, se les debe proponer que eliminen el tabaco fumado y el vapeo, se debe alentar a los consumidores a utilizar sustitutos de la nicotina fumada (ej., parches o chicles) y medicamentos contra el síndrome de abstinencia como el bupropión. Entre los fumadores, dejar de fumar reduce los problemas cardiovasculares y otros problemas de salud (Mons et al., 2015) lo que aumenta las probabilidades de supervivencia al coronnavirus tal y como muestran los datos del Centro Chino para el Control y la Prevención de Enfermedades los cuales revelan que el COVID-19 tiene una tasa de letalidad del 6,3\% para las personas con enfermedad respiratoria crónica, en comparación con el 2,3\% de la población general (Wu y McGoogan, 2020). Esta propuesta es muy similar a la planteada por Yafai, Sherry, Etengoff y Stuart (2020), quienes recomiendan a los consumidores de cannabis que deben limitar su consumo por vía fumada e inhalatoria y cambiar a comestibles, aceites y tinturas (comience con poco y vaya despacio); a pesar de que no hay datos claros acerca de que estas formas de consumo sean mejores o peores para las personas infectadas, parece lógico pensar que fumar podría agregar irritación a un pulmón infectado, por lo tanto, si está infectado o corre el riesgo de estarlo por COVID-19 abandone el consumo de cannabis.

Uno de los últimos estudios difundidos por estos colectivos tratan de presentar a la cannabis sativa (concretamente extractos con alto contenido en CBD) como una sustancia más efectiva en la prevención y tratamiento del COVID-19 que la Hydroxicloroquina, señalando los efectos pernicio- sos de esta última; no obstante, se trata de un estudio preprint sobre el uso de cannabis con un alto contenido de CBD como hipótesis para tratar los procesos inflamatorios del COVID, de momento en modelos de tejidos 3D humanos artificiales de vía oral, vía aérea e intestinal, concluyendo que al no ser concluyentes los resultados, se precisan más estudios (Wang, 2020).

Esta sobrecarga informativa engañosa o infoxicación sobre los supuestos beneficios terapéuticos del cannabis llega a la ciudadanía a través de internet, principalmente páginas webs que en la mayoría de las ocasiones son dominios propios de lo que denominamos «movimientos procannábicos», financiados directa o indirectamente por la industria cannábica como por ejemplo «Israel inicia pruebas clinicas para tratar el COVID-19 con cannabis» (www.canamo. net) y cuyo objetivo principal es crear en las redes sociales un estado de opinión favorable hacia el consumo del cannabis y su legalización (Isorna, Vázquez, Redondo y Veiga, 2019), lo cual repercutirá finalmente en las actitudes y comportamientos de las personas fuera de la red y por ende en la sociedad en general.

\section{A modo de conclusión}

Teniendo en cuenta la información disponible, es pertinente advertir que las personas que fuman cannabis y los usuarios que «vapean» sus derivados, tendrán riesgos mayores en la pandemia por COVID-19, riesgos que se suman a los numerosos ya conocidos del consumo del cannabis (García-Álvarez, Gomar, García-Portilla y Bobes, 2019); por lo tanto, dejar de fumar cannabis se convierte en una medida preventiva relevante para defenderse mejor del COVID-19.

La presente revisión, refuerza el convencimiento que no hay ninguna justificación científica para el uso del cannabis en ninguno de los síntomas o complicaciones producida por el virus SARS-CoV-2 (COVID-19), ni mucho menos para su prevención.

\section{Conflicto de Intereses}

Los autores declaran no tener ningún conflicto potencial de intereses.

\section{Bibliografía}

Agoritsas, T., Boroli, F., Calmy, A., Gartner, B., Gayetageron, A., Guessous, I.,... Zanella, M. (Groupe Guidelines COVID). (2020). Cannabinoïdes et COVID-19: évaluation pharmacologique. Hospitaux Universitaires Genève. Département de Médicine Aigüe. Service de Pharmacologie et Toxicologie Cliniques. Recuperado de https:// www.hug-ge.ch/sites/interhug/files/structures/coronavirus/documents/cannabinoides_et_covid-19.pdf. 
Blachman-Braun, R., Del Mazo-Rodríguez, R. L., López-Sámano, G. y Buendía-Roldán, I. (2014). Hookah, is it really harmless? Respiratory Medicine, 108, 661-667. doi:10.1016/j.rmed.2014.01.013.

Brown, J. D. (2020). Cannabidiol as prophylaxis for SARSCoV-2 and COVID-19? Unfounded claims versus potential risks of medications during the pandemic. Research in Social $\mathcal{E} \mathcal{F}^{2}$ Administrative Pharmacy. Avance de publicación on-line. doi:10.1016 / j.sapharm.2020.03.020.

Confac (2020). Nacida de la necesidad: se publica guía de buenas prácticas para el desconfinamiento de las asociaciones cannábicas. Recuperado de: https://confac.org/.

Dunlop, A., Lokuge, B., Masters, D., Sequeira, M., Saul, P., Dunlop, G., Ryan, J., Hall, M. y Maher, L. (2020). Challenges in maintaining treatment services for people who use drugs during the COVID-19 pandemic. Harm Reduction Journal, 17, 26. doi:10.1186/s12954-02000370-7.

Etemadi, A., Gandomkar, A., Freedman, N. D., Moghadami, M., Fattahi, M. R., Poustchi, H.,... Malekzadeh, R. (2017). The association between waterpipe smoking and gastroesophageal reflux disease. International Journal of Epidemiology, 46, 1968-1977. doi:10.1093/ije/dyx158.

Filbey, F. M., McQueeny, T., DeWitt, S. J. y Mishra, V. (2015). Preliminary findings demonstrating latent effects of early adolescent marijuana use onset on cortical archi-tecture. Developmental Cognitive Neuroscience, 16, 16-22. doi:10.1016/j.dcn.2015.10.001.

Galindo, C., González, A., Espigares, E. y Moreno, E. (2019). Riesgo para la salud pública de fumar tabaco en cachimba. Higiene y Sanidad Ambiental, 19, 1775-1785.

García, F. (9 de mayo de 2020). La marihuana como servicio esencial. La Vanguardia, pp.43.

García Álvarez, L., Gomar, J. J., García-Portilla, M. P. y Bobes, J. (2019). Cannabis use and cognitive impairment in schizophrenia and first-episode psychosis. Adicciones, 31, 89-94. doi:10.20882/adicciones.1328.

García-Álvarez, L., de la Fuente-Tomás, L., Sáiz, P. A., García-Portilla, M. P. y Bobes, J. (2020). Will changes in alcohol and tobacco use be seen during the COVID-19 lockdown?. Adicciones, 32, 85-89. doi:10.20882/adicciones. 1546 .

Hall, W., Degenhardt, L. y Teesson, M. (2009). Understanding comorbidity between substance use, anxiety and affective disorders: Broadening the research base. Addictive Behaviors, 34, 795-799. doi:10.1016/j.addbeh.2009.03.040.

Isorna, M., Vázquez, M. J., Redondo, L. y Veiga, S. (2019). Cómo pueden las fake news o falsas creencias contribuir al consumo de cannabis. En M. Isorna y A. Rial (Coord.) El consumo de cannabis y sus derivados: Mitos, posverdades y desafios (pp. 161-175). Madrid: Dykinson.

Israel inicia pruebas clínicas para tratar el COVID-19 con cannabis (s.f.). Recuperdo de https://canamo.net/ noticias/mundo/israel-inicia-pruebas-clinicas-para-tratar-el-covid-19-con-cannabis.

McCoy, K. L. (2016). Interaction between cannabinoid system and toll-like receptors controls inflammation. Mediators of Inflammation, ID 5831315. doi:10.1155/2016/5831315.

Mons, U., Müezzinler, A., Gellert, C., Schöttker, B., Abnet, C. C., Bobak, M., de Groot, L., ... CHANCES Consortium (2015). Impact of smoking and smoking cessation on cardiovascular events and mortality among older adults: meta-analysis of individual participant data from prospective cohort studies of the CHANCES consortium. The British Medical Journal, 350, h1551. doi:10.1136/bmj.h1551.

National Academies of Sciences, Engineering and Medicine. [NASEM]. (2017). The health effects of cannabis and cannabinoids: The current state of evidence and recommendations for research. Washington, DC: The National Academies Press. doi:10.17226/24625.

Owen, K. P., Sutter, M. E. y Albertson, T. E. (2014). Marijuana: respiratory tract effects. Clinical Reviews in Allergy $\mathcal{E}$ Immunology, 46, 65-81. doi:10.1007/s12016-013-8374-y.

Rial, A., Burkhart, G., Isorna, M., Barreiro, C., Varela, J. y Golpe, S. (2018). Cannabis use among adolescents: risk pattern, implications and possible explanatory variables. Adicciones, 31, 64-77. doi:10.20882/adicciones.1212.

Satre, D. D., Hirschtritt, M. E., Silverberg, M. J. y Sterling, S. A. (2020). Addressing problems with alcohol and other substances among older adults during the COVID-19 pandemic. The American Journal of Geriatric Psychiatry, S1064-7481(20)30296-7. Avance de publicación on-line. doi:10.1016/j.jagp.2020.04.012.

Schulte, M. T. y Hser, Y. (2013). Substance use and associated health conditions throughout the lifespan. Public Health Reviews, 35, 3. doi:10.1007/BF03391702.

Sexton, M. (2020). Cannabis in the time of coronavirus disease 2019: The yin and yang of the endocannabinoid system in immunocompetence. The Journal of Alternative and Complementary Medicine, 10, 1-5. doi:10.1089/ acm.2020.0144.

Sexton, M., Cudaback, E., Abdullah R., Finnell, J., Mischley, L., Rozga, M.,... Stella, N. (2014). Cannabis use by individuals with multiple sclerosis: effects on specific immune parameters. Inflammopharmacology, 22, 295-303. doi: $10.1007 /$ s10787-014-0214-z.

Sexton, M., Shelton, K., Haley P. y West, M. (2018). Evaluation of cannabinoid and terpenoid content: cannabis flower compared to supercritical CO2 concentrate. Planta Medica, 84, 234-241. doi:10.1055/s-0043-119361.

Tashkin, D. P. (2013). Effects of marijuana smoking on the lung. Annals of the American Thoracic Society, 10, 239-247. doi:10.1513/AnnalsATS.201212-127FR.

Tetrault, J. M., Crothers, K., Moore, B. A., Mehra, R., Concato, J. y Fiellin, D. A. (2007). Effects of marijuana smoking on pulmonary function and respiratory complica- 
tions: a systematic review. Archives of Internal Medicine, 167, 221-228. doi:10.1001/archinte.167.3.221.

Volkow, N. (2020). Collision of the COVID-19 and Addiction Epidemics. Annals of Internal Medicine. Avance de publicación on-line. doi:10.7326/M20-1212

Wang, B., Kovalchuk, A., Li, D., Ilnytskyy, Y., Kovalchuk, I. y Kovalchuk, O. (2020). In search of preventative strategies: novel anti-inflammatory high-CBD cannabis sativa extracts modulate ACE2 expression in COVID-19 gateway tissues. Preprints, 2020040315. doi:10.20944/ preprints202004.0315.v1.

Wu, L., Ghitza, U., Zhu, H., Spratt, S., Swartz, M. y Mannelli, P. (2018). Substance use disorders and medical comorbidities among high-need, high-risk patients with diabetes. Drug and Alcohol Dependence, 186, 86-93. doi:10.1016/j.drugalcdep.2018.01.008.

Wu, Z. y McGoogan, J.M. (2020). Characteristics of and important lessons from the Coronavirus Disease 2019 (COVID-19) outbreak in China: summary of a report of 72314 cases from the Chinese Center for Disease Control and Prevention. JAMA, 323, 1239-1242. doi:10.1001/ jama.2020.2648.

Yafai, S. y Etengoff, S. (2020). The case for cannabis. Advising cannabis users about COVID-19. Emergency Medicine News, 42, 5B. doi:10.1097/01.EEM.0000668076.42644.69. 